\title{
Hemispheric asymmetries for the temporal resolution of brief auditory stimuli
}

\author{
SUZANNE BROWN and MICHAEL E. R. NICHOLLS \\ University of Melbourne, Parkville, Victoria, Australia
}

\begin{abstract}
This study investigates a left-hemisphere ( $\mathrm{LH})$ advantage for the discrimination of fine temporal events within the auditory modality in 24 normal adults. Brief noise bursts were delivered monaurally to the left or right ears, half of which contained a gap lasting between 2 and 8 msec. Subjects were required to indicate whether or not the noise burst contained a gap. Research within this area has yielded conflicting results. It is possible that variations in gap position or the properties of the noise burst may have contributed to this conflict. The effect of gap position was investigated by systematically varying the position of the gap during half of the trials. White noise bursts were used to avoid the use of spectral cues. Reaction time, response bias, and response error measures revealed a right-ear (and hence LH) advantage for gap detection. Stable gap positions were recognized more accurately than variable gap positions. Gap position had no effect on the observed LH advantage. These results suggest that the auditory properties of noise burst, rather than gap position, play an important role in the discrepancies observed in this area.
\end{abstract}

Research into cerebral asymmetry has suggested that the left hemisphere $(\mathrm{LH})$ and the right hemisphere $(\mathrm{RH})$ of the brain have different capacities for the resolution of sensory information separated by fine moments of time. Some of the earliest research within this field was carried out by Efron (1963a, 1963b). Efron's experimental paradigm required neurologically normal subjects to judge whether two bilateral stimuli delivered to the left and to the right hands were simultaneous or successive. Subjects tended to judge the stimuli to be simultaneous when the stimulus to the left hand was delivered shortly before the stimulus to the right hand. Efron accounted for these results with a model of hemispheric asymmetry in which the LH was absolutely specialized for temporal processing. Performance asymmetries between the hands were believed to be the result of delays associated with the trans-callosal passage of information from the left hand. These findings have been supported by research conducted by Mills and Rollman (1980) within the auditory modality. Using a bilateral presentation procedure, they found that stimuli delivered to the left ear were perceptually delayed relative to stimuli delivered to the right ear during temporal-order judgments.

Asymmetries for temporal processing have also been observed in studies which have employed unilateral presentation procedures (wherein stimuli are presented to the same side within each trial). In research conducted within the visual modality, Nicholls (1994a) has found a right-visual-field advantage for the detection of two-

Robert Efron, Soren Buus, and an anonymous reviewer are thanked for their helpful comments on an earlier draft of this paper. Correspondence should be addressed to M. E. R. Nicholls, Department of Psychology, University of Melbourne, Parkville, VIC 3052, Australia (email: nicholls@psych.unimelb.edu.au). flash fusion and for the perception of simultaneity. Similar LH advantages have been reported for the auditory (Vroon, Timmers, \& Tempelaars, 1977) and tactile (Hammond, 1981) modalities. The absolute specialization model proposed by Efron (1963a, 1963b, 1963c) cannot account for the asymmetries found for unilateral presentations, because no transmission asynchronies are associated with the transfer of signals across the corpus callosum. An alternative model of hemispheric asymmetry, the relative superiority model, is better able to account for the functional asymmetries found in both unilateral and bilateral studies (Allen, 1983). This model assumes that information will be processed by the hemisphere that receives the information first. Thus, both hemispheres have some capacity for temporal resolution, although the LH is capable of making finer discriminations than is the RH.

Despite the evidence in favor of a temporal processing asymmetry, Clark and Geffen (1990) have recently questioned the reliability and generality of such an asymmetry in the LH. Their subjects were required to attend to pairs of vibratory stimuli delivered either unilaterally or bilaterally to the left or the right hand. The subjects were required to determine whether the onsets of the stimuli were simultaneous or successive. Using an adaptive staircase method to control the interval between the stimuli, Clark and Geffen found no evidence of a right-hand advantage. A study conducted by Efron, Yund, Nichols, and Crandall (1985) has failed to support the notion of a general LH superiority for temporal processing in the auditory modality. Each experimental trial consisted of two 300 -msec noise bursts, one of which contained a gap, presented in succession to either the left or the right ear. The subjects were required to indicate whether the first or the second noise burst contained the gap. Eight different gap 
durations, ranging from 2 to $7 \mathrm{msec}$, were presented. The results of this study revealed no evidence of a right-ear (and hence $\mathrm{LH}$ ) advantage.

Efron et al.'s (1985) results stand in direct contrast to those of Vroon et al. (1977). In that experiment, subjects were given monaural presentations of a $3-\mathrm{sec}$ noise burst that was continuous in some trials but that contained a brief interval (gap) in others. The duration of this interval was varied so that seven different gap durations, ranging from 1 to $4 \mathrm{msec}$ in half-millisecond steps, were presented randomly across trials. Results indicated a strong right-ear advantage for detection of the gap.

Efron et al. (1985) suggested that methodological factors accounted for the difference between their results and those of Vroon et al. (1977). Foremost among these was the location of the gap in the stimulus. Vroon et al. interrupted the 3 -sec stimulus at varying locations $(1.0,1.5$, or $2.0 \mathrm{sec}$ ) after onset, whereas the location of the gap in the study conducted by Efron et al. was always in the middle of the stimulus. Efron suggested that the significant rightear advantage found by Vroon et al. may have been related to temporal "uncertainty" surrounding the location of the gap. The way in which uncertainty of gap location may have facilitated right-ear performance was not discussed by Efron, and the reasoning behind this remains unclear. Indeed, one might have expected stimulus uncertainty to favor the functioning of the "holistic" RH rather than the "analytic" LH (Bradshaw \& Nettleton, 1983).

Alternatively, it is possible that the discrepancy between the results obtained by Efron et al. (1985) and Vroon et al. (1977) might have been related to the type of noise used. Efron et al. made use of narrow-band noise bursts containing frequencies in the range of $200-400 \mathrm{~Hz}$. Vroon et al. used white noise bursts that contained a far greater range of frequencies. Important differences exist between the temporal properties of the two types of noise. The long-term magnitude spectrum of white noise is not affected by brief interruptions (Moore, 1989). However, interruptions of narrow-band noise result in a spectral "splatter" outside the normal bandwidth of the sound (Buus \& Florentine, 1985). By monitoring differences in the spectral shapes of the sound, subjects are able to discriminate between gap and no-gap trials without using temporal cues. This problem can be overcome through the use of a band-stop masker, designed to mask the splatter (Moore, 1989). However, Efron et al. did not use a mask. As a result, subjects might have attended to the spectral shape of the sound rather than the temporal interruption associated with the gap. This phenomenon has been observed in an investigation of asymmetries for two-click fusion (Murphy \& Venables, 1970). A left-ear advantage was found for the detection of fusion between the clicks. Subjects reported using pitch cues to make their judgments rather than basing their decision on temporal information. The use of pitch cues would favor RH functioning (Gordon, 1970; Sidtis \& Bryden, 1978), possibly annulling or overriding any LH advantage.
The aim of the present study was to investigate the source of the discrepancy between the studies conducted by Efron et al. (1985) and Vroon et al. (1977). Subjects were required to make a forced two-choice discrimination related to the presence/absence of a gap in a burst of white noise. Gap location was systematically manipulated in half of the experimental trials (variable condition). In the remaining trials, the gap was located in the middle of the sound pulse (stable condition). If the right-ear advantage observed by Vroon et al. was the result of variability associated with the location of the gap, a right-ear advantage would be expected for the variable condition, but not for the stable condition. A finding of no change in the degree or direction of the asymmetry between the stable and variable conditions would suggest that gap location was not an important factor in the symmetry observed by Efron et al. The absence or presence of a rightear advantage across both conditions would provide an insight into the effects of noise type. The finding of a right-ear advantage in the stable and variable conditions would suggest that the white noise used in the present experiment (as opposed to the narrow-band noise used by Efron et al.) is needed in order for temporal processing asymmetries to emerge.

The performance measures were reaction time, response error, and response bias. In previous research, Nicholls (1994a) used similar measures to investigate visual field asymmetries for two-flash fusion. Faster reaction times and lower levels of error were found for right-visual-field presentations. Measures of response bias revealed higher rates of "no-gap" responses than of "gap" responses for trials directed to the RH. If the temporal processing capacity of the LH is supramodal, a similar set of results should be observed for auditory stimuli used in the present experiment. The demonstration of such an asymmetry would serve to reinforce the general case that the $\mathrm{LH}$ is specialized for the processing of temporal information and that this specialization is cross-modal.

\section{METHOD}

\section{Subjects}

Twenty-four subjects ( 14 male, 10 female) were recruited from the student population at the University of Melbourne. All subjects reported having normal hearing in both ears. Their ages ranged from 18 to 34 years $($ mean $=24.4$ years $)$. The subjects demonstrated a strong right-handed tendency (handedness quotient greater than or equal to +40 ) as measured by the Edinburgh Handedness Inventory (Oldfield, 1971).

\section{Apparatus}

The experiment was controlled with an IBM PC interfaced with a digital $/ \mathrm{O}$ card with an on-board millisecond timer (Blue Chip Technology, No. DCM-16). A red light-emitting diode (LED) mounted on a matte black display panel was used to warn the subject that a trial was about to begin. Auditory stimuli were generated via a gated white-noise generator connected to a set of headphones (Sennheiser HD40). The subjects' responses were recorded using a fourbutton response panel designed to allow a bimanual response with the index and middle fingers of each hand 


\section{Stimuli}

The auditory stimuli consisted of bursts of white noise delivered at an intensity of $74 \mathrm{~dB}$ SPL across the frequency spectrum. Two stimulus conditions were presented: (1) a continuous noise burst lasting 300-msec (no gap), and (2) a 300-msec noise burst interrupted by a brief cessation of the stimulus (gap). Gap location was manipulated by introducing an interruption centered at three different points within the noise burst: (1) early ( $75 \mathrm{msec})$, (2) middle $(150 \mathrm{msec})$, and ( 3 ) late $(225 \mathrm{msec})$. Four different gap durations were presented randomly across trials $(2.0,4.0,6.0$, and $8.0 \mathrm{msec}$ )

\section{Procedure}

A total of 480 trials were presented during each testing session. The total number of trials was divided into eight blocks of 60 trials. A five-level factorial design was used, with stimulus condition (gap, no gap), side of presentation (left ear, right ear), gap stability (stable, variable), gap location (early, middle, late), and gap duration (2, 4,6 , and $8 \mathrm{msec}$ ) as within-subjects factors. Gap stability and gap duration were varied between blocks. For half of the blocks, the position of the gap was stable and always appeared in the center of the white noise burst. In the remaining four blocks, the position of the gap was variable. In this case, the interruption in the gap trials occurred early, in the middle, or late within the burst of white noise. Equal proportions of the different gap locations occurred within each block, and these occurred in a random order. The four different gap durations were varied between blocks. Two blocks were devoted to each of the different gap durations. Gap placement was variable for one of these blocks and stable for the other. Within each block, stimulus condition and side of presentation were balanced and nested within one another. These parameters were then randomized to prevent subjects from predicting the position and type of the next trial.

Each subject attended one session of testing during which the handedness questionnaire was administered and the experimental task completed. The duration of the entire experimental session was approximately $45 \mathrm{~min}$. The experiment was conducted in a well-lit, soundproof room. Subjects were seated at a table with the response board and LED panel placed directly in front of them. They were informed that the trials would take one of two forms: one in which the noise burst was continuous, the other in which the noise was briefly interrupted by a gap in which there was no noise. To encourage subjects to adopt an unbiased criterion, they were told that $50 \%$ of the noise bursts would be continuous and $50 \%$ would contain a gap. They were also informed that the level of difficulty within each block would be consistent. The subjects were asked to indicate as quickly and as accurately as possible whether the trial was of a "gap" or "no-gap" type.

A bimanual response was used to avoid any effects related to motor biases between the hands. Half the subjects were instructed to use their middle fingers to indicate that the noise burst had been continuous, and to use their index fingers to signal the detection of a gap. The finger of response was reversed for the remaining subjects. To ensure that subjects were attending to the stimulus, the LED was activated $1 \mathrm{sec}$ prior to the beginning of each trial. Trials were separated by a 1.5 -sec interval. Trials in which subjects took longer than $2 \mathrm{sec}$ to respond were automatically rejected by the computer program and replaced with an identical trial at the end of the block.

Prior to commencement of the experimental trials, the subjects were given a number of practice trials to familiarize them with the equipment and the experimental procedure. Four blocks of 12 practice trials were presented. The practice blocks began at an easy level and became progressively more difficult, with gap durations of $100,20,10$, and $8 \mathrm{msec}$, respectively. The experimental trials commenced after each subject had reached a satisfactory level of response accuracy $(75 \%$ correct responses at a gap time of $8 \mathrm{msec}$ ).

\section{RESULTS}

The data were analyzed with a repeated measures analysis of variance, with side of presentation (right ear, left ear), gap stability (variable, stable), and gap duration $(2,4,6,8 \mathrm{msec})$ as within-subjects factors. The data were collapsed across the different gap locations (early, middle, late) for the variable trials and the two stimulus conditions (gap, no gap) for all trials.

\section{Response Error}

A measure of response error for each cell was calculated by summing the misses for the signal (gap) trials with false alarms for the noise (no-gap) trials, and then dividing by the total number of trials (30) and multiplying this figure by 100 . The error data are represented in Figure 1. Inspection of Figure 1 reveals a strong trend for error to rise as a function of the shorter gap durations, and this difference resulted in a significant main effect for gap duration $[F(3,69)=242.57, p<.001]$. Blocks associated with variable gap positions were associated with a significantly higher level of error than were blocks with a stable gap position $[F(1,23)=10.25, p<.01]$. Error rates for right-ear presentations were significantly lower than error rates on similar trials presented to the left ear $[F(1,23)=10.31, p<.01]$. The right-ear advantage was attenuated for gap durations of 2 and $8 \mathrm{msec}$ compared with gap durations of 4 and $6 \mathrm{msec}$, and this effect resulted in a significant interaction between gap duration and side of presentation $[F(3,69)=9.90, p<.001]$. No other interaction approached statistical significance.

\section{Reaction Time}

A measure of reaction time was calculated by averaging reaction times for correctly detected signal (gap) trials with reaction times for correctly detected noise (no-gap) trials. The reaction time data are represented in Figure 2.

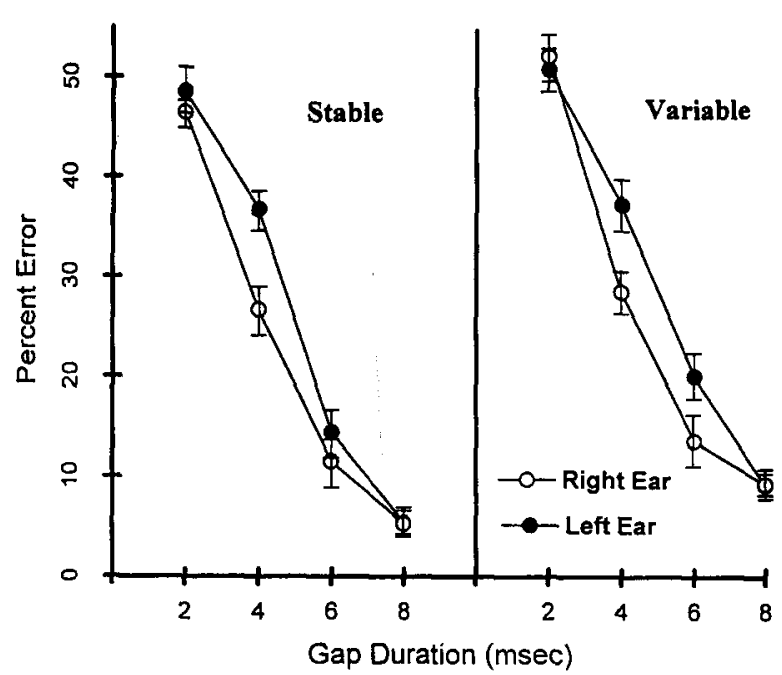

Figure 1. Mean percentage of response error $( \pm S E)$ for the right and left ears across the different gap durations in both stable and variable locations. 
Reaction times for the shorter gap durations were slower than those for the longer gap durations, and this resulted in a significant main effect for gap duration $[F(3,69)=$ $24.73, p<.001]$. Reaction times to trials presented to the right ear were significantly faster than those for trials presented to the left ear $[F(1,23)=4.3, p<.05]$. The effect of gap variability and all interactions were not significant.

\section{Response Bias}

Bias toward gap or no-gap responses was gauged by subtracting the number of "no-gap" responses from the number of "gap" responses (irrespective of whether they were correct). This measure of bias yielded a positive score if there was a high proportion of "gap" responses and a negative score if there was a high proportion of "nogap" responses. The response bias data are represented in Figure 3. There was a significant trend for the shorter gap durations to be associated with negative bias scores $[F(3,69)=33.22, p<.001]$. Figure 3 reveals a trend for left-ear presentations to be associated with negative bias scores (i.e., more "no-gap" responses were made) in comparison with similar right-ear presentations, and this difference resulted in a significant main effect of side of presentation $[F(1,23)=6.4, p<.05]$. The asymmetry between the ears was reduced for gap durations of 2 and $8 \mathrm{msec}$ in comparison with gap durations of 4 and $6 \mathrm{msec}$. This effect resulted in a significant interaction between gap duration and side of presentation $[F(3,69)=3.81$, $p<.05]$. The effect of gap variability and all other interactions were not significant.

\section{DISCUSSION}

Results indicated that right-ear responses were predominantly faster and more accurate than left-ear responses. Analysis of response bias demonstrated that subjects were much more likely to respond "no gap" on

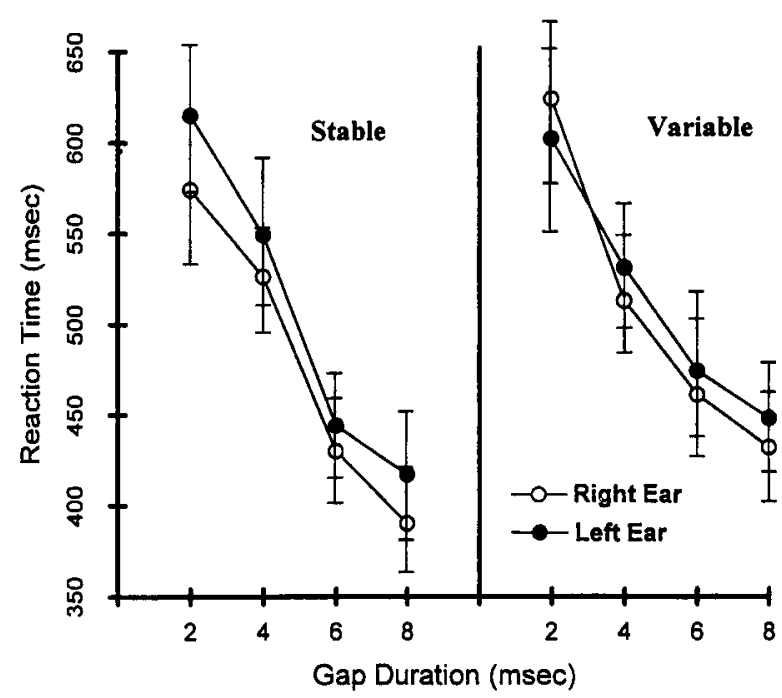

Figure 2. Mean reaction times $( \pm S E$ ) for the right and left ears as a function of gap duration and gap stability.

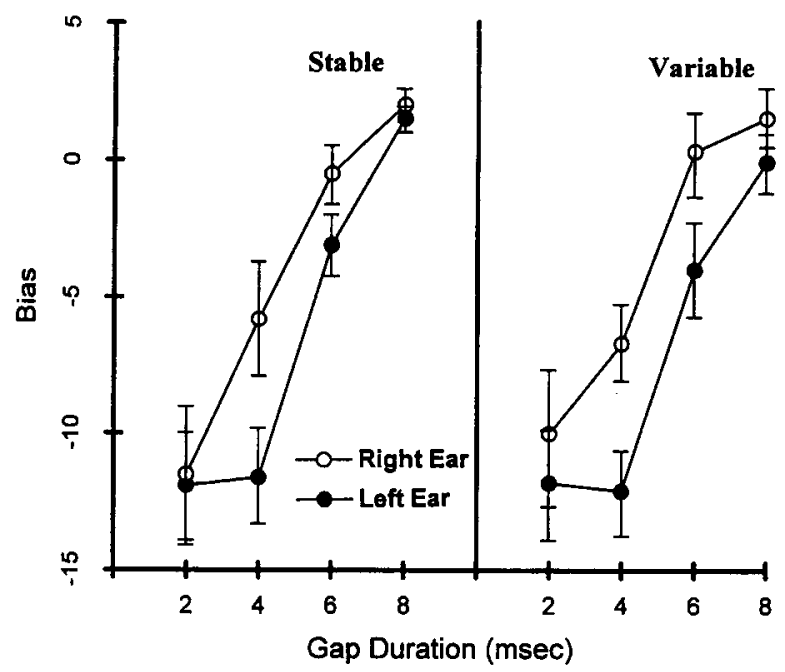

Figure 3. Mean response bias $( \pm S E$ ) for the right and left ears as a function of gap duration and gap stability. Positive scores indicate a tendency to respond "gap" more often, whereas negative scores indicate a tendency to respond "no gap" more often.

trials presented to the left ear as opposed to the right ear. The asymmetry for the error and response bias measures was marked for gap durations of 4 and $6 \mathrm{msec}$, but reduced for durations of 2 and $8 \mathrm{msec}$. Similar results, where the asymmetry is most pronounced in the middle of psychometric curve, have been reported by Nicholls (1994a) for the left and right visual fields in normal subjects and by Efron et al. (1985) for the hemispheres contralateral and ipsilateral to an anterior lobectomy in a clinical population. It is likely that the symmetry observed for the longer gap durations is the result of a ceiling effect in which both hemispheres are able to deal with a very simple task. Similarly, the symmetry observed for the shorter gap durations may be the result of a floor effect in which neither hemisphere is able to cope with a virtually impossible task. An LH advantage emerged only for the intermediate levels of the design, where performance was not constrained.

The current data provide support for the notion that the LH is capable of making finer temporal discriminations than is the RH. The ability to make such discriminations can be interpreted in terms of the amount of cortical neural noise that surrounds temporal events (Uttal, 1981). Thus, the critical gap duration represents the minimum time required for the neural response to the first stimulus to decay, before the onset of the second stimulus. If the time interval between the two events is decreased below this minimum time, then the probability that the two neural events will overlap will increase with an associated rise in the chance of perceptual fusion. The concept of neural noise is similar in essence to the decay of auditory sensation discussed by Plomp (1964). He suggested that temporal gaps within a signal were detected when the sensory activity associated with the gap fell below a certain ratio of the activity associated with the signal. Within this context, increased levels of neural noise 
could prolong the decay of the signal or add to the background levels of noise.

Bearing these models in mind, it is possible that the LH has a lower level of temporal noise than the RH (Nicholls, 1994a). The fact that the right ear was able to detect the gap at levels that were seemingly too brief to allow accurate detection for the left ear offers support for the view that the LH has a lower threshold of fusion. The high proportion of "no-gap" responses observed for left-ear presentations can be accounted for by high levels of temporal noise within the RH which obscure the gap, thus decreasing the likelihood of a gap response. It is worth noting that this response bias occurred despite subjects' being told that equal numbers of gap and no-gap trials would occur.

The fact that an asymmetry occurred under conditions of unilateral stimulus presentation, in which the effects of transcallosal transmission asynchronies are not involved, suggests that interhemispheric transfer time does not play an important role in cerebral asymmetries for temporal resolution. These findings cast doubt on the models that espouse exclusive specialization (Efron, 1963a, 1963b, 1963c; Mills \& Rollman, 1980). The present data more closely fit the assumptions of models that focus on the relative capacity of the hemispheres for specialized processing (Allen, 1983).

Analysis of the stable and variable gap conditions revealed higher levels of error for the variable gap condition. This finding is in accord with Mahneke (1958), who found that visual gap detection was most efficient when the gap was located within the middle of the stimulus pulse. No difference was observed across the stable and variable conditions for the reaction time and response bias measures. This lack of effect is most probably the result of combining the early and late conditions (in which performance is impaired) with the middle condition (in which performance is not impaired) for the variable data.

Examination of the asymmetry in the stable and variable conditions revealed a consistent right-ear advantage in both conditions. These results are not in accord with Efron et al. (1985), who predicted that a right-ear advantage would emerge only for the variable condition. Thus it would seem that unpredictable gap positions are not an important factor in the right-ear advantage observed by Vroon et al. (1977).

Why did Efron et al. (1985) fail to find a right-ear advantage? Their stimuli were similar in many respects to those in the present experiment. In both experiments, the duration of the stimulus pulse was $300 \mathrm{msec}$. Fifty percent of trials contained a gap, and $50 \%$ contained no gap. In both experiments, the duration of the gap ranged from approximately 2 to $8 \mathrm{msec}$, and this range covered floor to ceiling levels of performance (respectively). Efron et al. made use of a two-choice discrimination for pairs of stimuli with variable gap durations. In the present experiment, subjects were required to make a two-choice discrimination for individual stimuli in blocks in which gap duration was held constant. It is possible that this procedure allowed more stable levels of performance to be achieved than did the procedure used by Efron et al., in which task difficulty varied randomly.

A more important difference between the studies may be related to the auditory properties of the stimuli. Efron et al. (1985) used narrow-band noise, whereas broad-band white noise was used in the present experiment. Manipulation of gap stability had no effect on the right-ear advantage for the detection of gaps in broad-band noise. This suggests that the symmetry observed by Efron et al. is related to the narrow-band noise they used rather than gap stability. Previous research (Buus \& Florentine, 1985; Moore, 1989) has suggested that this type of noise needs to be masked to prevent the use of spectral cues. The lowpass narrow-band noise used by Efron et al. should also be associated with higher error rates for gap detection than those obtained with the broad-band noise in the present study. This is because the narrow-band noise filtered out the higher frequency components of the noise that provide more accurate temporal information. Despite this, remarkably similar error rates were observed in both studies for the different gap durations. It is likely that performance may have been enhanced in the present experiment by the presentation technique, which maintained constant levels of difficulty within blocks. This would have made the task easier, because subjects were able to adopt a constant criterion for the decisions within each block.

The present experiment provides strong support for the notion of an enhanced temporal processor contained within the LH. These results accord with the findings of Vroon et al. (1977) but conflict with the conclusions drawn by Efron et al. (1985). Given the weight of evidence from nonclinical (Efron 1963a, 1963b; Mills \& Rollman, 1980; Nicholls, 1994a, 1994b; Nicholls \& Atkinson, 1993; Nicholls \& Cooper, 1991; Robinson \& Solomon, 1974) and clinical research (Carmon, 1978; Carmon \& Nachshon, 1971; Goldman, Lodge, Hammer, Semmes, \& Mishkin, 1968; Lackner \& Teuber, 1973), it seems safe to conclude that an LH specialization for temporal processing does exist. The consistency of the LH advantage over a range of stimulus modalities suggests that the LH's enhanced capacity for temporal processing is crossmodal. The existence of a such a center provides an interesting insight into the functioning of the LH. It suggests that the cerebral hemispheres are specialized for low-level cognitive functions such as temporal resolution. Cerebral asymmetries at this level may play an important role in the development of other high-level functions, such as language (Tallal, Stark, \& Mellitis, 1985).

\section{REFERENCES}

Allen, M. (1983). Models of hemispheric specialization. Psychological Bulletin, 93, 73-104

Bradshaw, J. L., \& Nettleton, N. C. (1983). Human cerebral asymmetry. Englewood Cliffs, NJ: Prentice Hall.

Buus, A., \& Florentine, M. (1985). Gap detection in normal and impaired listeners: The effect of level and frequency. In A. Michelsen (Ed.), Time resolution in auditory systems ( $\mathrm{pp} .159-179)$. Berlin: Springer-Verlag. 
CARMON, A. (1978). Spatial and temporal factors in visual perception of patients with unilateral cerebral lesions. In M. Kinsbourne (Ed.), Asymmetrical functions of the brain (pp. 86-98). New York: Cambridge University Press.

CARMON, A., \& NACHSHON, I. (1971). Effects of unilateral brain damage on the perception of temporal order. Cortex, 7, 410-418.

Clark, C. R., \& GefFen, G. M. (1990). Hemispheric equivalence for simultaneity judgements of somatosensory stimuli. Cognitive Neuropsychology, 7, 311-327.

EFRON, R. (1963a). The effect of handedness on the perception of simultaneity and temporal order. Brain, 86, 261-283.

EFRON, R. (1963b). The effects of stimulus intensity on the perception of simultaneity in right- and left-handed subjects. Brain, 86, 285-294.

EFron, R. (1963c). Temporal perception, aphasia and déjà vu. Brain, 86, 403-424.

Efron, R., Yund, E. W., Nichols, D. R., \& Crandall, P. H. (1985). An ear asymmetry for gap detection following anterior temporal lobectomy. Neuropsychologia, 23, 43-50.

Goldman, P. S., Lodge, A., Hammer, L. R., Semmes, J., \& MishKIN, M. (1968). Critical flicker frequency after unilateral temporal lobectomy in man. Neuropsychologia, 6, 335-363.

Gordon, H. W. (1970). Hemispheric asymmetries in the perception of musical chords. Cortex, 6, 387-398.

Hammond, G. R. (1981). Finer temporal acuity for stimuli applied to the preferred hand. Neuropsychologia, 19, 325-329.

LACKNER, J. R., \& TEUBER, H.-L. (1973). Alterations in auditory fusion thresholds after cerebral injury in man. Neuropsychologia, 11, 409-415.

MAHNEKE, A. (1958). Foveal discrimination measured with two successive light flashes. Acta Ophthalmologica, 36, 3-11.

Mills, L., \& Rollman, G. B. (1980). Hemispheric asymmetry for auditory perception of temporal order. Neuropsychologia, 18, 4l-47.

MOORE, B. C. J. (1989). An introduction to the psychology of hearing. London: Academic Press.

Murphy, E., \& Venables, P. (1970). Ear asymmetry in the threshold of fusion of two clicks: A signal detection analysis. Quarterly Journal of Experimental Psychology, 22, 288-300.
Nicholls, M. E. R. (1994a). Hemispheric asymmetries for temporal resolution: A signal detection analysis of threshold and bias. Quarterly Journal of Experimental Psychology, 47A, 291-310.

NichOLLs, M. E. R. (1994b). The non-contribution of attentional biases to visual field asymmetries for temporal discrimination. Neuropsychologia, 32, 209-220.

Nicholls, M. E. R., \& AtKInson, J. (1993). Hemispheric asymmetries for an inspection time task: A general left hemisphere temporal advantage? Neuropsychologia, 31, 1181-1190.

NiChOLLS, M. E. R., \& COOPER, C. J. (1991). Hemispheric differences in the rates of information processing for simple non-verbal stimuli. Neuropsychologia, 29, 677-684.

OLDFIELD, R. C. (1971). The assessment and analysis of handedness: The Edinburgh inventory. Neuropsychologia, 9, 97-113.

Plomp, R. (1964). Rate of decay of auditory sensation. Journal of the Acoustical Society of America, 36, 277-282.

Robinson, G. M., \& Solomon, D. J. (1974). Rhythm is processed by the speech hemisphere. Journal of Experimental Psychology, 102, 508-511.

SiDTiS, J. J., \& BRyden, M. P. (1978). Asymmetrical perception of language and music: Evidence for independent processing strategies. Neuropsychologia, 16, 627-632.

Tallal, P., Stark, R., \& Mellitis, D. (1985). The relationship between auditory temporal analysis and receptive language development: Evidence from studies of developmental language disorder. Neuropsychologia, 23, 314-322.

UTTAL, W. R. (1981). A taxonomy of visual processes. Hillsdale, $\mathrm{NJ}$ : Erlbaum.

Vroon, P. A., Timmers, H., \& TempelaArs, S. (1977). On the hemispheric representation of time. In S. Dornic (Ed.), Attention and performance VI: Proceedings of the Sixth International Symposium on Attention and Performance (pp. 231-245). Hillsdale, NJ: Erlbaum.

(Manuscript received February 27, 1996; revision accepted for publication May 19, 1996.) 